\title{
TRABAJO DE CAMPO EN LA COSTA SUR DEL PERÚ
}

\author{
Dwight Wallace
}

El plan del Proyecto Arqueológico Fulbright ha sido trabajar en áreas no estudiadas de la Costa Peruana, a fin de relacionarlas con secuencias conocidas de las áreas costaneras del norte, centro y sur; de manera que puedan correlacionarse estas secuencias entre sí. El trabajo fue organizado a manera de un estudio de conjunto que delinearía las secuencias arqueológicas por medio de un trabajo más intensivo, incluyendo excavaciones.

Resolví comenzar el trabajo en el centro mismo de la costa sur-central y trabajar en dirección sur hacia el área Ica-Nasca. Esto fue previsto para coincidir con el trabajo de Stumer, que debería comenzar en el siguiente valle al Norte y continuar en la misma dirección hasta el valle del Rímac.

Por consiguiente, se comenzó el trabajo en el valle de Chincha continuando en Pisco el siguiente valle hacia el sur.

El único trabajo significativo previamente hecho en estos valles fue el de Max Uhle en Chincha, publicado en 1924 por Kroeber; y el de Engel en Pisco, publicado en American Antiquity en 1957. El trabajo de Uhle determinó los periodos de estilos cerámicos Inca y el inmediatamente anterior a éste (Chincha Tardío I) basado en agrupaciones de tumbas. En Pisco,
Engel encontró cerámica Paracas y Nascoide cerca del yacimiento arqueológico de Tambo Colorado. La arquitectura de este interesante yacimiento ha sido también descrita y fotografiada en las publicaciones de varios autores.

Con la ayuda de fotografías aéreas se hizo un reconocimiento sistemático de los yacimientos de Chincha y Pisco. Se localizaron 110 yacimientos arqueológicos en Chincha; y 104 en Pisco. Todos los datos relativos a estos yacimientos fueron apuntados en formularios preparados, las colecciones de fragmentos de cerámica y otros materiales pertinentes fueron hechos para cada yacimiento y los yacimientos mismos fueron ubicados en mapas a escala de 1/40000, mostrando las vías de acceso y otras informaciones para facilitar su reubicación. Estos mapas fueron especialmente dibujados de las fotografías aéreas, ya que no existían mapas apropiados a la escala necesaria.

A medida que progresaba el reconocimiento, se hizo evidente que las relaciones culturales de los valles de Chincha y Pisco se encontraban principalmente hacia el Sur, particularmente en el valle de Ica, Fue posible, por consiguiente, usar nuestros conocimientos sobre las relaciones cronológicas de la cerámica en Ica para poder agrupar los diferentes tipos de cerámica hallados en Chincha y Pisco, en su orden 
cronológico. Con esta secuencia basada en la cerámica, fue posible construir secuencias para métodos de construcción, patrones de poblamiento y otros rasgos de la arqueología.

Hasta este punto los conocimientos obtenidos sobre Chincha y Pisco, resultaron a menudo ser más completos, aunque menos detallados que los datos anteriores que se puedan obtener de los valles de Ica y Nasca. Por ello creí conveniente permanecer algún tiempo en Ica, ya que podía ampliar los datos requeridos. Este trabajo todavía continúa. Se han agregado 30 nuevos yacimientos a los aproximadamente 60 que habían sido anotados anteriormente por Rowe, Menzel, Strong y Dawson, los yacimientos más antiguos están en proceso de ser revisados nuevamente, si es necesario. Se han hecho formularios de informes y colecciones de fragmentos de cerámica para todos estos yacimientos, y han sido anotados en el mapa a escala $1 / 40000$ previamente dibujados por Rowe. El resultado será un juego de datos para Ica que es comparable al obtenido en Chincha y Pisco. Los datos y las colecciones estarán permanentemente accesibles en el Museo de la Universidad Nacional Mayor de San Marcos en Lima.

Además del trabajo de reconocimiento, se han hecho algunas excavaciones con el objeto de aclarar interrogantes de importancia que el reconocimiento dio a luz. Dos excavaciones fueron hechas en Chincha y una está todavía en realización en Ica. Una en cada valle. Es un corte estratigráfico de basurales, la tercera, en Chincha, resultó no tener una estratigrafía cultural, pero si ha brindado una excelente muestra de cerámica. Todas las excavaciones están hechas en basurales de Paracas.

Para delinear las secuencias de cerámica del área, la más temprana conocida hasta ahora ha sido encontrada en las excavaciones hechas en Chincha y en Ica. La decoración consiste en dibujos geométricos simples en incisiones poco profundas, círculos estampados e incisos, líneas cóncavas estampadas en forma zonada (zoned rocker stamping) y también dibujos geométricos simples en pintura postcocción. Las vasijas están cocidas a fuego bajo y las superficies son negras o marrón oscuro. Una forma decorada característica, es una va- sija de costados rectos, con bordes inclinados y/o ligeramente ensanchados. Las unidades de estos dos yacimientos comparten muchos rasgos, pero también difieren en muchos otros. El estilo general tiene muchas similitudes con las cerámica chavinoide de la costa central. Las unidades de Ica carecen de incisiones en zigzag estampadas (rocker stamping), pero en cambio tiene dibujos en negativo y la combinación de pintura e incisión del estilo Paracas. Por consiguiente, está más cercano a dicho estilo y en realidad yace directamente debajo de los basurales de Paracas Temprano; e indicaría que se trata del antecedente del estilo Paracas.

Para el estilo Paracas, hay una colección proveniente tanto de Chincha como de Pisco, que es suficientemente grande, de manera que se puede establecer una comparación con las diferentes fases que han sido distinguidas dentro del estilo Paracas en Ica. Las dos unidades son generalmente similares; no es exactamente igual a la cerámica Paracas de Ica, pero tipológicamente podrían ser seriadas entre Paracas Temprano y Paracas Tardío. Alternativamente podrían representar una variación local. Una de las características notables es la frecuente combinación del negativo o inciso con pintura post-cocción en la misma vasija.

En el periodo siguiente, equivalente al Proto Nasca de Strong o al Nasca I de Dawson, parece haber habido diferentes locales entre las áreas de Nasca-Ica y Chincha-Pisco. En la última ocurre un estilo que consiste principalmente en una cerámica delgada, pulida de color anaranjado y sin decoración. Los tipos relativamente raros con decoración incluyen bruñido de molde, engobe crema, puntuación interior y ocasionalmente un engobado rojo e inciso exterior. Se pueden aislar dos fases dentro del estilo. Han sido denominados Chungos A y B, de la localidad tipo en el valle de Pisco. Las características principales que distinguen a estas dos fases, es la ocurrencia de dos tipos de vasijas en Chungos B, una vasija o plato de bordes en ángulo.

Chungos A comparte muchas características con el estilo Jahuay, descubierto por Lanning a $20 \mathrm{~km}$ al Norte de Chincha, cuando puede ser convenientemente definido, Chungos A parece ser idéntico al estilo Jahuay. 
Chungos B está aislado en varios yacimientos del valle de Pisco y puede ser bien definido. El estilo Chungos en general, es similar a lo que se conoce como estilo Necrópolis. Como se ha definido el estilo ocurre también en el valle de Chincha, asimismo ocurre en Ica pero allí está asociado a tipo engobado con decoración incisa en el contorno, característico del tipo Nasca I y Proto Nasca de Dawson y Strong respectivamente, así como otros tipos decorados, no encontrados en Pisco o en Chincha.

El siguiente estilo de cerámica encontrado en Chincha y Pisco es policroma, de estilo engobado. El yacimiento tipo es El Carmen, en Chincha. El rasgo más característico son líneas diagonales de color rojo y blanco, alternado en una base negra en los exteriores de un plato con paredes en ángulo, bajas (gambreled). Un tipo de decoración muy similar por su forma al Chungos B con el exterior completamente negro y el interior completamente rojo, (solid black...) es temprano dentro del estilo en general; puede probar que sea exclusivamente anterior a la cerámica con dibujos pintados o alternativamente, asociado con el estilo policromo en sus fases más tempranas. La decoración en este estilo incluye dibujos geométricos policromados y dibujos representativos, en negro, rojo, púrpura, rojo naranja y algunas veces gris en un fondo negro. Vasijas de tipo abierto son las formas decoradas más comunes. El estilo tiene varios rasgos similares con la cerámica de Ica y Nasca, específicamente con Nasca 2 de Dawson.

El rasgo más característico de el estilo siguiente: La Estrella, son vasijas abiertas con líneas diagonales de color blanco alternando con negro con un fondo oxidado, no engobado, en los exteriores. Los interiores de las vasijas de esta forma y las superficies decoradas de las otras formas de este tipo tienen un engobe blanco, con dibujos geométricos y dibujos representativos en negro, rojo púrpura, rojo naranja y algunas veces dibujos de pigmentos grises. Zonas con engobe rojo sin dibujos también ocurres. Este estilo comparte varios rasgos con los estilos Nasca Temprano y Nasca Medio de Strong y también con Nasca 3 y 4 de Dawson.

Para las fases posteriores del estilo Nasca la cerámica de Pisco y Chincha parece ser muy similar a la encontrada en Ica y Nasca.
En general, los rasgos locales que distinguen los estilos El Carmen y La Estrella de las fases correspondientes del estilo Nasca, se encuentran con mayor fuerza en Chincha, ocurren con bastante frecuencia en Pisco, son raros en Ica, y puede no ocurrir en absoluto en Nasca. Contrariamente, rasgos que caracterizan al estilo Nasca tal como es encontrado en Nasca e Ica, se encuentran más frecuentemente en Pisco que en Chincha. Por añadidura, las similitudes se vuelven más específicas y más frecuentes en Chincha y Pisco, en las fases tardías del estilo Nasca.

El Periodo Medio, en cierto modo, parece corresponden a una laguna, tanto en el valle de Chincha como en el de Pisco. Uhle recolectó un poco de cerámica de estilo Wari puro, en la parte alta del valle de Pisco y Lanning ha encontrado algunas tumbas del mismo estilo en Jahuay. A pesar de ello, no se ha encontrado arquitectura o restos de habitación que pudieran ser identificados con este periodo. Los únicos fragmentos de cerámica pertenecientes a este periodo que fueron encontrados en ambos valles son unos fragmentos Epigonales de unas tumbas removidas en Chincha. Este vacío puede ser explicado, por lo menos en parte, por la existencia de algún factor que pudiera ser difícil la localización e identificación de los yacimientos de este periodo. Sin embargo, la reciente teoría de Lanning y Menzel de que habían dos centros distintos de influencia Wari, en Pachacamac y en Nasca, sugiere que la situación se puede interpretar como evidenciado una ligera influencia en las áreas intermedias, que incluirían Chincha y Pisco. Es significativo anotar que la falta de influencia Wari ha sido también encontrada en Cañete, el valle siguiente al Norte de Chincha.

Sin embargo, hay algunas indicaciones de que un estilo local relacionado existió en el área durante ese periodo.

En los periodos Intermedio Tardío, Tardío y Colonial Temprano, la cerámica de Chincha y Pisco es distinguible. La secuencia en Pisco sigue a la de Ica, sin diferencias locales, excepto alguna marcada influencia de Chincha, que la que se encuentra en Ica. Esta secuencia incluye los estilos Chulpaca, Soniche, Tacaraca A y Tacaraca B identificados por Menzel (ms). En 
Chincha la situación es menos clara. Las tumbas Chulpaca y Soniche existen en Chincha, pero tampoco en la cantidad o asociaciones que podrían indicar que estos fueron estilos dominantes en el valle. Sin embargo, los estilos locales correspondientes no han sido todavía claramente identificados, desde que las colecciones de superficie para este periodo son pocas y en pequeña cantidad. En contraste, están los lotes de tumbas excavados por Uhle, así como varias colecciones superficiales de fragmentos de cerámica, de las cuales se pueden definir los estilos más recientes. El primero de estos, el Chincha Tardío de Kroeber y Strong, ha sido colocado por Menzel como contemporáneo con una fase de estilo transicional entre los estilos de Soniche y Tacaraca A y por ello inmediatamente preinca en fecha. El estilo muestra fuertes influencias tanto de Ica como de Cañete, pero también tienen ciertos rasgos propios. El estilo de cerámica siguiente, del periodo de la ocupación Inca del Valle, es distinta del estilo previo y también de los estilos contemporáneos de los valles adyacentes. Aparentemente este estilo persistió en los principios del periodo Colonial Chincha. Fragmentos de cerámica de trueque de Inca, de estos estilos Tacaraca A (Inca) y Tacaraca B (resurgimiento local, post-conquista) ocurren también con alguna frecuencia.

Para volver a otros materiales referiremos que una secuencia de construcción de muros de adobe ha sido estudiada para Chincha, en base a la cerámica asociada. Hay un tipo de adobe, que llamo tipo "cuña horizontal", que presenta la forma de un grano de maíz y descansa de costado con una parte plana hacia afuera y está asociado con la cerámica de estilo Paracas. Es usado para cubrir paredes, con un relleno de terrones de adobe de tamaño de una toronja a un melón. El siguiente tipo es también en forma de cuña, pero más aplanado, largo, ancho y colocado verticalmente, descansando en un costado de tope aplanado -el tipo "cuña vertical"-. Es usado en masas sólidas, no exactamente para cubrir paredes como el tipo anterior. Aparentemente distinto y posterior en tiempo que el adobe tipo "cuña vertical”, es el adobe hemisférico; usado con la base hacia abajo y solamente en paredes sin techo. Este está definitivamente asociado con la cerámica de estilo El Carmen. Siguiendo a este tipo hay una forma semicilíndrica (de base rectangular, la parte superior curva, extremos semicirculares) que, con varios subtipos, es la firma asociada con los varios subestilos tardíos de Nasca, hasta el fin de este estilo general. La construcción en el periodo Intermedio Tardío es de tapia, barro sólido, probablemente en forma vaciada. Los adobes vuelven a aparecer en asociación con la ocupación Inca, de forma rectangular grande, hecho con molde.

Más o menos la misma secuencia de adobes se aplica al valle de Pisco. Por lo menos se han encontrado los tipos hemisféricos y semicilíndricos, asociados a la misma cerámica que en Chincha. Ha sido más difícil observar la forma de adobe en Pisco, dada la naturaleza y condición de los yacimientos. Pero también se observa que hubo un mayor uso de la piedra, particularmente en las áreas en que las hay en abundancia. Esto es especialmente verídico para los yacimientos de Chungos y Nasca Temprano; además, uno de los principales yacimientos Inca en el valle, está construido con piedras del campo. Una nueva clase de adobe fue observada en Pisco, de forma larga y cónica, variando de 1 a 2 pies de longitud, descansando con el extremo aplanado hacia la cara de la pared.

La secuencia a base de formas de adobe en Chincha es en general similar a lo que se conoce hasta la fecha en Inca; algunas formas son idénticas, otras solo análogas. Las asociaciones con la cerámica son generalmente las mismas, con respecto a este periodo.

Son de gran interés los tipos de estructura para estos métodos de construcción y los patrones generales de población, para los cuales estos métodos de construcción son usados. Los tipos de adobe de "cuña horizontal" y terrones irregulares ocurren en grandes montículos sólidos, de los cuales hay cinco en Chincha, todos orientados en una línea Este-Oeste y con la misma forma. El fechado de estos yacimientos no puede ser posterior a la cerámica de estilo Chungos y más parece ser Paracas. Los montículos con indudablemente función ceremonia; las habitaciones parecen haber sido bastante pequeñas, la población dispersa. En Chincha el tipo de adobe de "cuña vertical" es usado para un tipo idéntico de montículo ceremonial 
al mencionado anteriormente, y probablemente pertenezca aproximadamente al periodo de la cerámica estilo Chungos. Sin embargo, el yacimiento tipo de Chungos que está situado en Pisco, consiste de dos grandes estructuras rectangulares, subdivididos uniformemente en recintos rectangulares y demasiado grandes para ser llamados cuartos.

Las dos estructuras están construidas sobre suaves elevaciones, y la depresión entre forma más o menos una plaza. El yacimiento fue, obviamente, el resultado de un planeamiento completo, y ha sido probablemente un centro ceremonial o administrativo. Hay un sitio similar de este periodo en Cabeza Larga, cerca del yacimiento de Paracas Cavernas, son restos de habitaciones, pero las estructuras son más pequeñas y de un planeamiento menos regular que el yacimiento de Chungos.

Asociada con la cerámica de estilo El Carmen, hay poblaciones bastante grandes que consisten de cuartos contiguos de varias formas y tamaños; no se distinguen calles rectas, pero las ciudades tenían una o más plazas entre ellas. Hay tres de estas ciudades en Chincha y Pisco, para las cuales los planos están todavía muy bien preservados.

Ningún yacimiento que pueda clasificarse como ceremonial ha sido identificado para este periodo, ni tampoco hay muchos para las siguientes fases de Nasca. En suma, los yacimientos de estas fases son difíciles de interpretar con respecto a su función y tipo de población.

En el periodo Intermedio Tardío el tipo básico es un montículo levantado en varias fases sucesivas de construcción, alcanzando los mismos, alturas considerables. Estos montículos están cubiertos con habitaciones, terrazas y patios, distribuidos más o menos al azar. Ocurren aislados (en este caso, son generalmente de tamaño pequeño) y en grupos de tres o cuatro. Además, hay cinco grupos separados en Chincha en número de veinte o más montículos. La disposición de estos montículos no tiene un orden discernible, y pueden estar uno al lado del otro o distanciados entre sí, a distancias variables. Generalmente un montículo puede ser identificado como un centro ceremonial especial o un centro administrativo. En suma, se puede clasificar a este tipo de yacimiento como un centro urbano, junto con las ciudades Nasca más tempranas.

Este patrón general persistió durante el periodo Colonial Temprano y Tardío. Solo dos sitios se pueden clasificar como de construcción pura o mayormente Inca: Tambo Colorado y Lima La Vieja, ambas en el valle de Pisco. En Chincha, las construcciones Inca se sumaron a los yacimientos existentes, incluyendo un "palacio" edificado en una de las plataformas del montículo ceremonial de La Centinela, que es parte de un centro urbano. Un sistema de carreteras conectaba los dos centros de administración Inca en el valle, incluyendo una que llevaba directamente al centro Inca en el valle de Pisco.

Un detalle adicional de interés es que existen frisos de adobe en algunos yacimientos del periodo Intermedio Tardío. Son muy similares a aquellas de la costa Norte en técnica y dibujos geométricos que llenas la superficie, pero los motivos específicos son diferentes.

Los aportes que el trabajo hecho en los valles de Chincha, Pisco e Inca han dado a la historia general de la cultura del área de la Costa Sur, son de considerable interés. Para resaltar lo más importante, la identificación de la cerámica más temprana conocida, relacionándola cercanamente al Chavinoide de la Costa Central y anterior al estilo local de Paracas, indican que las influencias que dominaron gran parte del Perú en este periodo, también se extendieron hasta la Costa Sur, con fuerza considerable e indudablemente formaron parte del desarrollo de los posteriores. Parece haber habido una mayor uniformidad aún, en el desarrollo cultural del Perú que el que se había sospechado.

La mayoría de los estilos posteriores fueron básicamente locales, pero, comenzando con el estilo Paracas, el grado de localidad no fue muy restringido. En la parte restante del periodo Temprano y en todo el periodo Intermedio Temprano, la uniformidad de la cerámica y otros patrones culturales se extendió a lo largo de una gran área, comprendiendo los valles de Chincha, Pisco, Ica, Nasca y, para el estilo Nasca, Acarí. Además, la existencia de grandes estructuras ceremoniales que datan 
desde temprano en este periodo, implican un sistema social y religioso más organizado que el que previamente se había pensado. Siguiendo a éstas, la presencia de centros urbanos en el periodo Intermedio Temprano contradice la teoría de que este tipo de población fue introducido en la Costa en una fecha posterior; estas son las poblaciones más tempranas en el Perú que caben dentro de las clasificaciones de centros urbanos (específicamente establecimientos urbanos), y ocurren desde Chincha hasta Acarí.

Parece haber habido menos independencia local dentro del área de la Costa Sur en el periodo Intermedio Temprano que en el periodo Intermedio Tardío, para el cual tenemos evidencias históricas de que Chincha, Ica (junto con Pisco) y Nasca fueron políticamente independientes. En el periodo Intermedio Tardío, el cuadro para toda el área es de rasgos locales distinguibles, además de una gran cantidad de interinfluencias. La influencia del estilo de cerámica Inca fue un fuerte factor unificador en la cerámica de toda el área, pero, por lo menos en la parte más reciente de este periodo, había una influencia de aproximadamente igual fuerza en Chincha, proveniente del valle de Cañete hacia el norte. Por ende, junto con sus rasgos locales, el valle de Chincha tiene una cierta independencia en este periodo. Sin embargo, hay una unidad básica dentro del área, y, excluyendo las influencias a lo largo de toda la Costa que se difundieron por toda ellas en los periodo Medio y Tardío, las influencias externas de áreas restringidas y cercanamente adyacentes. Por lo tanto, hoy se puede considerar, con base, a toda el área entre Chincha y Acarí, como una unidad geográfica que guardó una independencia básica y una uniformidad a través de su historia.

Tanto el Informe sobre el Reconocimiento del valle de Chincha como el presente Informe Preliminar, presentados por el Dr. Dwight T. Wallace, corresponden al Proyecto Arqueológico auspiciado por la Comisión Fulbright y dirigido por el Instituto de Arqueología y Etnología de la Universidad Nacional Mayor de San Marcos en 1957 y 1959. 